\title{
THE PERFEGT AND EXTREME SENARY FORMS
}

\author{
E. S. BARNES
}

This paper is devoted partly to a summary of some results which will be published in detail elsewhere, ${ }^{1}$ partly to a description of a new extreme senary form, $\phi_{6}$, and an $n$-dimensional generalization of it.

The main result is the determination of all classes of extreme senary forms. There are just six classes, represented by:

$$
\begin{aligned}
& \phi_{0}=\sum_{1}^{6} x_{i}^{2}+\sum_{i<j} x_{i} x_{j}, \\
& \phi_{1}=\phi_{0}-x_{1} x_{2}, \\
& \phi_{2}=\phi_{0}-x_{1} x_{2}-x_{1} x_{3}, \\
& \phi_{3}=\phi_{0}-\frac{1}{2}\left(x_{1} x_{2}+x_{3} x_{4}+x_{5} x_{6}\right), \\
& \phi_{4}=\phi_{0}-\frac{1}{2}\left(x_{1} x_{2}+x_{3} x_{4}+x_{3} x_{5}+x_{3} x_{6}+x_{4} x_{5}+x_{4} x_{6}+x_{5} x_{6}\right), \\
& \phi_{6}=\phi_{0}-\frac{1}{2}\left(2 x_{1} x_{2}+x_{1} x_{3}+x_{1} x_{6}+x_{2} x_{5}+x_{4} x_{6}+2 x_{5} x_{6}\right) .
\end{aligned}
$$

Each of these forms has minimum $M=1$ for integral $\mathbf{x} \neq \mathbf{0}$. They are listed in the following table, together with the symbol used by Coxeter (3), the order of the group $\mathfrak{g}$ of automorphs, the number $s$ of pairs of minimal vectors, and the value of $2^{6} \mathrm{D} / \mathrm{M}^{6}$ in decreasing order of this quantity.

\begin{tabular}{ccccc}
\hline Form & $\begin{array}{c}\text { Coxeter's } \\
\text { symbol }\end{array}$ & Order of $\mathfrak{g}$ & $s$ & $2^{6} D / M^{6}$ \\
\hline$\phi_{0}$ & $A_{6}$ & $2.7 !$ & 21 & 7 \\
$\phi_{3}$ & & 96 & 21 & $13.3^{3} / 2^{6}$ \\
$\phi_{6}$ & & 672 & 21 & $7^{3} / 2^{6}$ \\
$\phi_{1}$ & $B_{6} \sim D_{6}$ & $2^{6} .6 !$ & 30 & 4 \\
$\phi_{4}$ & $E_{6}{ }^{3}$ & $144.6 !$ & 27 & $3^{5} / 2^{6}$ \\
$\phi_{2}$ & $E_{6}$ & $144.6 !$ & 36 & 3 \\
\hline
\end{tabular}

Thus there is just one class of absolutely extreme forms, represented by $\phi_{2}$; and, in agreement with Blichfeldt (2),

$$
\gamma_{6}{ }^{6}=\max _{f} \frac{M^{6}}{D}=\frac{1}{D\left(\phi_{2}\right)}=\frac{64}{3} .
$$

Received March 7, 1956; in revised form October 16, 1956.

${ }^{1}$ The complete enumeration of extreme senary forms, Phil. Trans. Roy. Soc. London, $A$ 249 (1957), 461-506. 
The method used to establish these forms is the algorithm for perfect forms given by Voronoi (6). Hence all classes of perfect senary forms are now determined; these are represented by the extreme forms $\phi_{0}, \ldots, \phi_{4}, \phi_{6}$ and

$$
\phi_{5}=\phi_{0}-\frac{1}{2}\left(x_{1} x_{2}+x_{3} x_{4}+x_{3} x_{5}+x_{4} x_{5}\right) .
$$

The forms $\phi_{0}, \phi_{1}, \phi_{2}$ and $\phi_{4}$ were found by Coxeter (3), who gives a full description of their properties. Previously, both Voronoi (6) and Hofreiter (4) had established $\phi_{0}, \phi_{1}$ and $\phi_{2}$ (this being Voronoi's notation for them). As is pointed out by Coxeter (3), Hofreiter's list contains a fourth form, $F_{4}$, which is not extreme, but which is presumably an arithmetical slip for $^{2} \phi_{3}$.

The form $\phi_{3}$ was recently discovered independently by Kneser (5) and the author (1), and the reader is referred to these articles for a full description of its properties.

The remaining extreme form $\phi_{6}$ and the perfect non-extreme form $\phi_{5}$ are new. Apart from its existence as the simplest known form of its type, $\phi_{5}$ has little structural interest; we merely note that it has

$$
M=1, \quad D=3^{4} / 2^{10}, \quad s=22,
$$

and that its group $\mathfrak{g}$ is of order 288 .

On applying to $\phi_{6}(\mathbf{x})$ the transformation

$$
\mathbf{x}=\frac{1}{7}\left(\begin{array}{rrrrrr}
3 & 6 & 2 & 5 & 1 & 4 \\
5 & 3 & 1 & 6 & 4 & 2 \\
4 & 1 & 5 & 2 & -1 & 3 \\
-4 & -1 & 2 & -2 & 1 & -3 \\
-1 & -2 & -3 & -4 & 2 & 1 \\
-6 & -5 & -4 & -3 & -2 & -1
\end{array}\right) \mathbf{y}
$$

we obtain

$$
4 \phi_{6}(\mathbf{x})=2 \sum_{1}^{6} y_{i}^{2}+2 \sum_{i<j} y_{i} y_{j}=2 \phi_{0}(\mathbf{y})
$$

or, defining $y_{7}$ by the relation

$$
\begin{aligned}
\sum_{1}^{7} y_{i} & =0 \\
4 \phi_{6}(\mathbf{x}) & =\sum_{1}^{7} y_{i}^{2} .
\end{aligned}
$$

Since $2 \phi_{0}$ has determinant 7 , and the transformation (1) has determinant $1 / 7$, it follows that $D\left(\phi_{6}\right)=7^{3} / 2^{12}$. It is also easily verified from (1) that $\mathbf{x}$ is integral if and only if $\mathbf{y}$ is integral and satisfies

$$
\sum_{1}^{7} i y_{i} \equiv 0(\bmod 7)
$$

${ }^{2} \mathrm{As}$ Professor Coxeter has remarked to me, the correct value of $2^{6} \mathrm{D} / \mathrm{M}^{6}$ for $\phi_{3}$ is obtained by replacing 53 by 52 in $F_{4}$. 
Thus $\phi_{6}$ now appears as the case $n=6$ of the form $f_{n}$ defined, in terms of its lattice, by

$$
f_{n}=\sum_{1}^{m} y_{i}^{2}
$$

where the $y_{i}$ take integral values subject to

$$
\begin{gathered}
\sum_{1}^{m} y_{i}=0, \\
\sum_{1}^{m} i y_{i} \equiv 0(\bmod m)
\end{gathered}
$$

(where we have set $m=n+1$ for convenience). This form is extreme for all $n \geqslant 6$, but the proof of this for odd $n$ is rather involved. In what follows we shall restrict ourselves to even $n$ (odd $m$ ).

Let $\mathbf{e}_{1}, \ldots, \mathbf{e}_{m}$ denote the unit vectors in $m$-space, with coordinates $y_{1}, \ldots, y_{m}$. Now $f_{n}$ takes only even values subject to (7). It takes the value 2 only when $\mathbf{y}=\mathbf{e}_{i}-\mathbf{e}_{j}$, and clearly none of these sets satisfies (8). It takes the value 4 when

$$
\mathbf{y}=\mathbf{e}_{a}+\mathbf{e}_{b}-\mathbf{e}_{c}-\mathbf{e}_{d}
$$

with

$$
a+b \equiv c+d(\bmod m) .
$$

Thus $M\left(f_{n}\right)=4$. Since the lattice defined by (7), (8) has determinant $m^{\frac{1}{2}} . m$, we have $D\left(f_{n}\right)=m^{3} ;$ so

$$
(2 / M)^{n} D=(n+1)^{3} / 2^{n} .
$$

The number of pairs of minimal vectors is ${ }^{3}$

$$
s=\frac{1}{8} n(n+1)(n-2)
$$

For, for any $a, b$, the number of distinct unordered pairs $c, d$ satisfying (10) is $\frac{1}{2}(m-1)$, of which one pair is $a, b$. Since $a, b$ may be chosen in $\frac{1}{2} m(m-1)$ ways, this gives $2 s=\frac{1}{2} m(m-1) \frac{1}{2}(m-3)$, as asserted.

We now show that $f_{n}$ is eutactic. Regarding $y_{m}$ as the redundant variable in (6), we have

$$
f_{n}=\left(\sum_{1}^{n} y_{i}\right)^{2}+\sum_{1}^{n} y_{i}^{2}
$$

with adjoint

$$
F_{n}=n \sum_{1}^{n} y_{i}^{2}-2 \sum_{i<j} y_{i} y_{j} .
$$

The linear forms associated with the minimal vectors (9) are then

$$
\lambda_{k}=y_{a}+y_{b}-y_{c}-y_{d} \quad(k=1, \ldots, s),
$$

${ }^{3}$ For odd $n, s=\frac{1}{8}(n-1)^{2}(n+1)$. 
with $y_{m}=0$. We now see that

$$
\frac{1}{2}(m-3) F_{n}=\sum_{1}^{s} \lambda_{k}^{2} .
$$

For, by symmetry, each $y_{i}$ occurs in $4 s / m=\frac{1}{2}(m-1)(m-3)$ forms $\lambda_{k}$, giving a coefficient $\frac{1}{2}(m-1)(m-3)$ of $y_{i}{ }^{2}$ in $\sum \lambda_{k}{ }^{2}$. A pair $y_{i}, y_{j}$ occurs with like signs in $\frac{1}{2}(m-3)$ forms (as above); and with opposite signs in $m-3$ forms, this being the number of solutions of $d-b \equiv i-j$ with $d, b$ distinct from $i$ and $j$. This gives a coefficient $m-3-2(m-3)=-(m-3)$ of $y_{i} y_{j}$ in $\sum \lambda_{k}{ }^{2}$. Collecting these results and setting $y_{m}=0$, we obtain (12).

To prove that $f_{n}$ is perfect, we begin by defining the vectors

$$
\mathbf{f}_{i}=\mathbf{e}_{i}+\mathbf{e}_{i+1}-\mathbf{e}_{i+2}-\mathbf{e}_{i-1} \quad(i=1, \ldots, m),
$$

where all suffixes and congruences are to be interpreted modulo $m$. Then

$$
\sum_{1}^{m} \mathbf{f}_{i}=0
$$

but any $m-1$ of the $\mathbf{f}_{i}$ are independent. Hence we may take a new coordinate system $z_{1}, \ldots, z_{n}$ defined by

$$
\mathbf{z}=\sum_{1}^{n} z_{i} \mathbf{f}_{i}
$$

We have now to show that if all minimal vectors $\mathbf{z}$ of $f_{n}$ satisfy a quadratic relation

$$
p(\mathbf{z})=\sum_{1}^{n} p_{i j} z_{i} z_{j}=0,
$$

then all $p_{i j}=0$. Using (12), we shall express some of the minimal vectors in terms of $\mathbf{f}_{1}, \ldots, \mathbf{f}_{m}$; if $\mathbf{f}_{m}$ occurs in the expression, we must of course replace it by

$$
-\sum_{1}^{n} \mathbf{f}_{i}
$$

before using (14).

(i) Suppose first that $m \geqslant 9$ (and odd). We use in (14) the minimal vectors

$$
\begin{array}{r}
\mathbf{f}_{i}+\mathbf{f}_{i+1}+\ldots+\mathbf{f}_{i+r}=\mathbf{e}_{i+r}+\mathbf{e}_{i+1}-\mathbf{e}_{i+r+2}-\mathbf{e}_{i-1} \\
(r \not \equiv \pm 1,-3) \\
\mathbf{f}_{i}+\mathbf{f}_{i+2}+\ldots+\mathbf{f}_{i+2 r}=\mathbf{e}_{i}+\mathbf{e}_{i+2 r+1}-\mathbf{e}_{i+2 r+2}-\mathbf{e}_{i-1} \\
(2 r \not \equiv-1,-2,-3),
\end{array}
$$

the given conditions ensuring that the suffixes on the right are distinct. Values of $i$ giving a suffix $0(\bmod m)$ on the left will not be considered.

We obtain at once from (15), with $r=0$,

$$
p\left(\mathbf{f}_{i}\right)=p_{i i}=0 .
$$


Using (15) with indices $r, r-1$, and subtracting, we obtain

$$
2 p_{i, i+r}+2 p_{i+1, i+r}+\ldots+2 p_{i+r-1, i+r}+p_{i+r, i+r}=0 ;
$$

hence, from (18),

$$
p_{i, i+r}+p_{i+1, i+r}+\ldots+p_{i+r-1, i+r}=0 \quad(r=3, \ldots, m-4) .
$$

Replacing $i, r$ by $i+1, r-1$ and subtracting from (19) gives

$$
p_{i, i+r}=0 \quad(r=4, \ldots, m-4) .
$$

From (16) with $r=1$, and $p_{i i}=0$,

$$
p_{i, i+2}=0, \quad p_{1, m-1}=0 ;
$$

from (15) with $r=2$ and (21),

$$
p_{i, i+1}+p_{i+1, i+2}=0 \text {. }
$$

From (19) with $r=3$ and (21)

$$
p_{i, i+3}+p_{i+2, i+3}=0 \text {. }
$$

We now use (17), and (15) with $r=m-6$; subtracting the results gives

$$
p_{i-3, i}+p_{i-3, i+1}+\ldots+p_{i-3, i+m-6}=0 \text {. }
$$

This holds certainly for $i=4$ and $i=1$ (no suffix then being zero), whence

$$
\begin{aligned}
& p_{14}+p_{15}+\ldots+p_{1, m-2}=0, \\
& p_{1, m-2}+p_{2, m-2}+\ldots+p_{m-5, m-2}=0 .
\end{aligned}
$$

By (20), these reduce to

whence

$$
p_{14}+p_{1, m-2}=p_{1, m-2}+p_{m-5, m-2}=0,
$$

$$
p_{14}=p_{m-5, m-2} .
$$

By (23) with $i=1$ and $i=m-5$, this gives

But (22) gives

$$
p_{34}=p_{m-3, m-2} \text {. }
$$

$$
p_{34}=-p_{45}=p_{56}=\ldots=-p_{m-3, m-2},
$$

and so, with (24),

$$
p_{34}=p_{m-3, m-2}=0 .
$$

Now (22) and (23) give at once

$$
p_{i, i+1}=0, \quad p_{i, i+3}=0 .
$$

This, with (18), (20) and (21), establishes that all $p_{i j}=0$ except possibly $p_{1, m-2}$ and $p_{2, m-1}$. That these are also zero now follows immediately by using the minimal vectors (from (15) with $r=1$ ) 


$$
\begin{aligned}
-\left(\mathbf{f}_{m}+\mathbf{f}_{2}\right) & =\mathbf{f}_{1}+\mathbf{f}_{3}+\mathbf{f}_{4}+\ldots+\mathbf{f}_{m-1}, \\
-\left(\mathbf{f}_{m-2}+\mathbf{f}_{m}\right) & =\mathbf{f}_{1}+\mathbf{f}_{2}+\ldots+\mathbf{f}_{m-3}+\mathbf{f}_{m-1} .
\end{aligned}
$$

Thus all $p_{i j}=0$, and so $f_{n}$ is perfect.

(ii) When $m=7,(17)$ is no longer a minimal vector and the above analysis fails. All 21 vectors are now

$$
\mathbf{f}_{i}, \mathbf{f}_{i}+\mathbf{f}_{i+2}, \mathbf{f}_{i}+\mathbf{f}_{i+1}+\mathbf{f}_{i+2} \quad(i=1, \ldots, 7),
$$

and a simple calculation establishes the perfection of $f_{n}=\phi_{6}$.

It is easy to verify that $\mathbf{f}_{1}, \ldots, \mathbf{f}_{m-1}$ give not only a basis of the space, but also a basis for the lattice of $f_{n}$. Hence we find the cyclically symmetrical representation

$$
\left.f_{n}(\mathbf{x})=\sum_{1}^{m}\left(x_{i}+x_{i-1}-x_{i-2}-x_{i+1}\right)^{2} \quad \text { ( } n \text { even }\right),
$$

with $x_{m}=0$, the suffixes being taken modulo $m$. A similar representation exists also for $n$ odd.

We now consider the group $g$ of automorphs of $\phi_{6}$; we denote by (s) the contragadient group of automorphs of its adjoint $F_{6}$. Since $\mathfrak{g}$ must permute the minimal vectors (more precisely, the pairs $\mathbf{\pm} \mathbf{m}$ of minimal vectors), (5) permutes the associated linear forms. From (11), any permutation of the $\lambda_{k}$ leaves $F_{6}$ invariant. It follows that $(5)$ is precisely the group of linear transformations permuting the associated linear forms.

From this result, we shall show that (5) (and so $\mathrm{g}$ ) has order 672, is transitive on the linear forms, ${ }^{4}$ and is isomorphic to $\mathscr{E}_{2} \times P G L(2,7)$. Corresponding to the representation (25), we may write the associated linear forms (11) as

where

$$
z_{i}, z_{i}+z_{i+2}, z_{i}+z_{i+1}+z_{i+2} \quad(i=1, \ldots, 7),
$$

$$
\begin{gathered}
z_{i}=y_{i}+y_{i+1}-y_{i+2}-y_{i-1}, \\
\sum_{1}^{m} z_{i}=0 .
\end{gathered}
$$

Obvious elements of (S) are $\mathrm{S}: y_{i} \rightarrow y_{i+1}$ and $\mathrm{U}: y_{i} \rightarrow y_{3 i}$ (where the suffixes are taken modulo 7 ). These show that $(\xi)$ is transitive on the linear forms. For $\mathrm{S} z_{i}=z_{i+1}$, so that the forms of each set $\left(z_{i}\right),\left(z_{i}+z_{i+2}\right),\left(z_{i}+z_{i+1}+z_{i+2}\right)$ are permuted cyclically by $\mathrm{S}$; while

$$
\begin{aligned}
& \mathrm{U} z_{7}=y_{7}+y_{3}-y_{6}-y_{4}=z_{7}+z_{2} \\
& \mathrm{U}^{2} z_{7}=y_{7}+y_{2}-y_{4}-y_{5}=-\left(z_{3}+z_{4}+z_{5}\right) .
\end{aligned}
$$

Let now $(\$)_{7}$ be the subgroup of (5) which leaves $z_{7}$ invariant (or changes its sign). We shall say that two of the linear forms combine if their sum or difference is an associated linear form. This property being invariant under (5), $\$_{7}$ must permute the 8 forms combining with $z_{7}$, namely

$$
\begin{array}{llllll}
z_{1}+z_{2}+z_{3}, \quad-z_{5}-z_{7}, & z_{6}+z_{1}, & -z_{7}-z_{2}, \\
& z_{4}+z_{5}+z_{6}, \quad z_{5}, & -z_{6}-z_{7}-z_{1}, \quad z_{2} .
\end{array}
$$

${ }^{4}$ Contrary to what one might expect from (12), (\$) is no longer transitive for $n>6$. 
Using the element $\mathrm{T}$ of $\mathrm{BH}_{7}$, of order 8 , defined by

$$
\begin{aligned}
\mathrm{T}\left(z_{1}, \ldots, z_{7}\right)= & \left(-z_{7}-z_{1}-z_{2},\right. \\
& \left.z_{1}+z_{2}+z_{3},-z_{3}-z_{5}, z_{5}+z_{6}+z_{7},-z_{6}-z_{7}-z_{1}, z_{1}, z_{7}\right)
\end{aligned}
$$

we see that $\mathrm{BF}_{7}$, considered as a permutation group on the set (26), is transitive on this set. The successive elements $\mathrm{T}^{n} z_{2}(n=1, \ldots, 8)$ are in fact the 8 elements (26), in the order written.

Let then $\left(S_{7,2}\right.$ be the subgroup of $\left(\xi_{7}\right.$ which leaves $z_{2}$ invariant. (We note that $\left(S_{7,2}\right.$ must leave the relative signs of $z_{7}, z_{2}$ unaltered, since $z_{7}+z_{2}$ is a linear form, while $z_{7}-z_{2}$ is not.) $\$_{7,2}$ must permute the 2 forms $z_{6}+z_{7}+z_{1}$, $z_{1}+z_{2}+z_{3}$ which combine with both $z_{7}$ and $z_{2}$. A direct argument now shows that $B_{7,2}$ is of order 4 and consists of the elements $\pm I, \pm V$, where

$$
\mathrm{V}\left(z_{1}, \ldots, z_{7}\right)=\left(-z_{7}-z_{1}-z_{2}, z_{2},-z_{6},-z_{2}-z_{4},-z_{5}-z_{7},-z_{3}, z_{7}\right) .
$$

It follows that (5) has order $21.8 .4=672$, as asserted, and is generated by S, T, U, V and - I. Since

$$
\mathrm{V}=-\mathrm{S}^{3} \mathrm{~T}^{5} \mathrm{~S}, \quad \mathrm{U}=-\mathrm{T}^{6} \mathrm{~S}^{5} \mathrm{~T}^{7},
$$

we see that $B=\{S, T,-I\}=\{-I\} \times\{S, T\}$.

We may identify $\{\mathrm{S}, \mathrm{T}\}$ with $P G L(2,7)$, consisting of the regular $2 \times 2$ matrices over $G F(7)$ (where multiples of the same matrix are identified), by means of the mapping

$$
\begin{aligned}
& \mathrm{S} \leftrightarrow \sigma=\left(\begin{array}{lr}
1 & 1 \\
0 & 1
\end{array}\right), \\
& \mathrm{T} \leftrightarrow \tau=\left(\begin{array}{rr}
1 & -2 \\
2 & 1
\end{array}\right) .
\end{aligned}
$$

For, as is well known, $\operatorname{PSL}(2,7)$ (consisting of those elements of $P G L(2,7)$ whose determinant is a square) is a simple group of order 168 generated by $\sigma$ and

$$
\theta=\tau^{4}=\left(\begin{array}{rr}
0 & -1 \\
1 & 0
\end{array}\right),
$$

and may be defined by the relations

$$
\sigma^{7}=\theta^{2}=(\sigma \theta)^{3}=\left(\sigma^{4} \theta\right)^{4}=1 .
$$

Now $\tau$ has determinant 5 , a non-square, so that $\sigma, \tau$ generate $P G L(2,7)$; we may take the further defining relation as

$$
\tau^{-1} \sigma \tau=\sigma^{4} \theta \sigma^{2} \theta \sigma^{5} .
$$

A simple calculation shows that the relations (27), (28) are satisfied if $\sigma, \tau, \theta$ are replaced by $\mathrm{S}, \mathrm{T}, \mathrm{T}^{4}$ respectively. We note that, under the given mapping, the elements of $\operatorname{PSL}(2,7)$ correspond to the elements of (5) of determinant +1 (expressed as integral unimodular transformations on, say, $z_{1}, \ldots, z_{6}$ ). 
We consider finally a conjecture made by Coxeter (3, p. 392), that every eutactic form with $s \geqslant \frac{1}{2} n(n+1)$ is perfect, and so extreme (the condition $s \geqslant \frac{1}{2} n(n+1)$ being of course necessary for perfection). We can use the form $f_{n}$ considered above to show that the conjecture is unfortunately false.

Let $f\left(x_{1}, \ldots, x_{n}\right)$ be any eutactic form with $s \geqslant \frac{1}{2} n(2 n+1) ; f_{n}$ is such a form for even $n \geqslant 10$ (in fact for all $n \geqslant 10$ ). Define

$$
g\left(x_{1}, \ldots, x_{2 n}\right)=f\left(x_{1}, \ldots, x_{n}\right)+f\left(x_{n+1}, \ldots, x_{2 n}\right) .
$$

Then $M(g)=M(f)$, and is attained when and only when one $f$ is zero and the other assumes its minimum. Hence the number of pairs of minimal vectors of $g$ is $2 s \geqslant \frac{1}{2} .2 n(2 n+1)$. Since $f$ is eutactic and the adjoint of $g$ is a multiple of $F\left(x_{1}, \ldots, x_{n}\right)+F\left(x_{n+1}, \ldots, x_{2 n}\right), g$ is clearly eutactic. But $g$ is not perfect, since it is disconnected: all minimal vectors satisfy, for example, the relation $x_{1} x_{n+1}=0$.

I am indebted to Dr. G. E. Wall for helpful discussions on the group of $\phi_{6}$.

\section{REFERENCES}

1. E. S. Barnes, Note on extreme forms, Can. J. Math. 7 (1955), 150-154.

2. H. F. Blichfeldt, The minimum values of positive quadratic forms in six, seven and eight variables, Math. Z. 39 (1935), 1-15.

3. H. S. M. Coxeter, Extreme forms, Can. J. Math. 3 (1951), 391-441.

4. N. Hofreiter, Ueber Extremformen, Monatsh. Math. Phys. 40 (1933) 129-152.

5. M. Kneser, Two remarks on extreme forms, Can. J. Math. 7 (1955), 145-149.

6. G. Voronoi, Sur quelques propriétés des formes quadratiques positives parfaites, J. reine angew. Math. 183 (1908), 97-178.

\section{University of Sydney}

